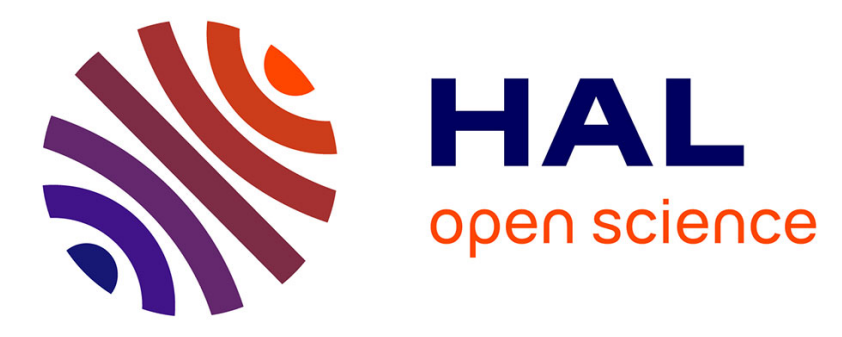

\title{
Review of integrated vehicle dynamics control architectures
}

Moad Kissai, Bruno Monsuez, Adriana Tapus

\section{To cite this version:}

Moad Kissai, Bruno Monsuez, Adriana Tapus. Review of integrated vehicle dynamics control architectures. 2017 European Conference on Mobile Robots (ECMR), Sep 2017, Paris, France. 10.1109/ECMR.2017.8098687 . hal-01690788

\section{HAL Id: hal-01690788 \\ https://hal.science/hal-01690788}

Submitted on 23 Jan 2018

HAL is a multi-disciplinary open access archive for the deposit and dissemination of scientific research documents, whether they are published or not. The documents may come from teaching and research institutions in France or abroad, or from public or private research centers.
L'archive ouverte pluridisciplinaire HAL, est destinée au dépôt et à la diffusion de documents scientifiques de niveau recherche, publiés ou non, émanant des établissements d'enseignement et de recherche français ou étrangers, des laboratoires publics ou privés. 


\title{
Review of Integrated Vehicle Dynamics Control Architectures
}

\author{
Moad Kissai, Bruno Monsuez, Adriana Tapus \\ ENSTA ParisTech \\ Department of Computer and System Engineering \\ 828 Boulevard des Marchaux, \\ 91762 Palaiseau Cedex, France \\ E-mail: \{moad.kissai, bruno.monsuez, adriana.tapus\}@ensta-paristech.fr
}

\begin{abstract}
Most of the chassis systems are developed by automotive suppliers to improve a specific vehicle performance. Drivers, and consequently vehicle manufacturers, are more concerned by the overall behaviour of the Vehicle. Many coordination architectures have been proposed in the literature in order to integrate different chassis systems in a single vehicle. In this paper, these architectures are compared and discussed. Two major classes are proposed: Downstream and Upstream Coordination. The purpose of this classification is to help car manufacturers and suppliers standardize an Integrated Vehicle Dynamics Control architecture for faster and more flexible designs.

Index Terms-Control Architectures, Chassis Systems, Systems Coordination, Vehicle Dynamics Control, Control Allocation.
\end{abstract}

\section{INTRODUCTION}

In today's automotive sector, driving experience, vehicle safety, and environmental protection are key competition criteria between automakers. In this context, Original Equipment Manufacturers (OEMs) offer constantly new attractive subsystems. Advances in automotive Electronic Control Units (ECUs), sensors, and actuators make the cost of vehicle embedded systems constantly decreasing. The number of chassis control systems grows rapidly as well as the number of competing systems in a single vehicle [1].

However, each vehicle subsystem has an independent control logic to accomplish a specific objective. These objectives compete and may implement contradictory logic: if a brakebased yaw control is used to reduce oversteer or understeer, the longitudinal acceleration demand in a cornering operation will deteriorate. Since new hardware is always expensive, improvements must be provided by the synergies that already exist between subsystems [2]. If instead of implementing competing systems, subsystems get coordinated, over-actuation would offer new opportunities to improve the overall system's safety and performance [3]. This could be done by developing a supervised overall vehicle control system, where information is shared by many subsystems, less resources are required, and computational costs are decreased therefore avoiding unnecessary duplication [2]. This has been given the name of interoperability in [4].

This will influence the vehicle's overall behavior. Thus, the high-level control architecture should be designed by car manufacturers. As different suppliers are concerned, a standardization of actuators, sensors, and software components interfaces is crucial. The development of this architecture requires a close collaboration between vehicle manufacturers and their suppliers. Therefore, any proposed solution should respect the intellectual property rights (IPR) of both sides [5]. In this context, three essential capabilities have been cited in [2]:

- Adaptability and dynamic reconfiguration to face environment changes, drivers behaviors, and failures [6],

- Plug-and-play extensibility to rapidly insert additional technologies without redesigning the whole architecture,

- Openness to support various systems from different sources.

The main purpose of this work is to propose a new classification of integrated control architectures to help designers choose a combination approach for a specific need or for the overall system. This would not be the first try, as in [4] a classification of integrated control methodologies for road vehicles has been made. The architectures have been differentiated as centralized, supervisory, hierarchical, and coordinated control. However, no discussion is provided so as to assign an architecture to a specific goal. Another recent classification was proposed in [7], where the classification has been simplified to affect the architectures to the desired goals. Two classes have been proposed: single-criterion and multicriterion integration motion control. In the single-criterion integration motion control approach, the integrated systems are combined to improve one single aspect of the vehicle. In the multi-criterion integration motion control approach, the combined systems aim to manage different concurrent vehicle dynamics. However, for each class, we could find different combination methodologies depending on the problem's complexity.

A main difference if not the main difference that we distinguished from the different architectures reviewed, is the position of the coordination layer in the control flow [8]. Two main approaches could be adopted to deal with the subsystems combination problem: either we treat the interactions and the eventual conflicts after the subsystems' operations or we control the commands distribution to the different subsystems in order to generate the desired synergies and avoid conflicts. We call the first approach systems downstream coordination, 
and the second, systems upstream coordination.

We start with a presentation of the downstream coordination approach in Section II and we explain why it was preferred by auto-makers until these days. We then give a presentation of the upstream approach and justify why it should be investigated and replace the downstream coordination approach (see Section III). In Section IV, we propose a general architecture where the different researches seem to converge to and we discuss the open challenges arising from its use. Finally, conclusion is be drawn and some future works are outlined (see Section V).

\section{SYSTEMS DOWNSTREAM COORDINATION}

We choose the appellation "systems downstream coordination" because this approach consists of studying the standalone chassis systems interactions after the commands generation by these subsystems. The coordination is made downstream. To schematize this approach, in Fig. 1 we reproduce the simple structure given by [8], where this approach is called the "bottom-up approach".

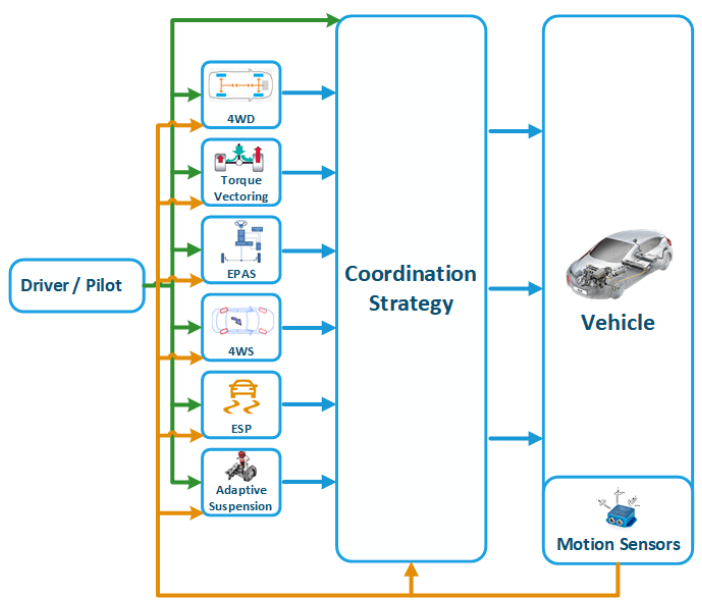

Fig. 1. Structure of the downstream coordination approach (adapted from [8]).

In [4] and [9], this is rather called a decentralized control. It follows a parallel structure where each subsystem works separately. Controllers have to work simultaneously. They have their own information system and Electronic Control Unit (ECU), which require more costs and space [10]. The only way to add "integration" in the process is by allowing additional communications between the controllers as the Fig. 2 shows.

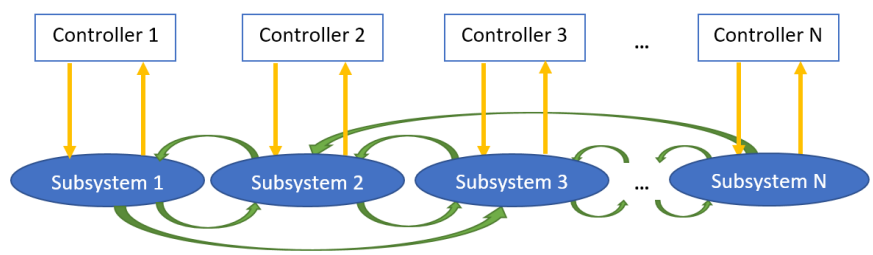

Fig. 2. Decentralized control structure (adapted from [4]).

This architecture is more suited for some manufacturing systems [9]. where it is called heterarchical. The fact that there is no global supervision makes this architecture particularly less suitable for vehicle safety control.

In order to develop a coordination strategy, interactions between the subsystems are studied. Automakers engineers use their "expert knowledge" to develop handling controllers. This is achieved through a rule-based controllers arbitration deduced from the subsystems interactions studies [8]. In this context, four coordinator types have been distinguished in [4]: Pure Subsumption, Largest Modulus Activation, Artificial Neural Network, and Fuzzy Logic Control.

\section{A. Pure Subsumption}

Regarding the pure subsumption approach, the highest level non-zero command takes precedence over of the other subcommands (see Fig. 3).

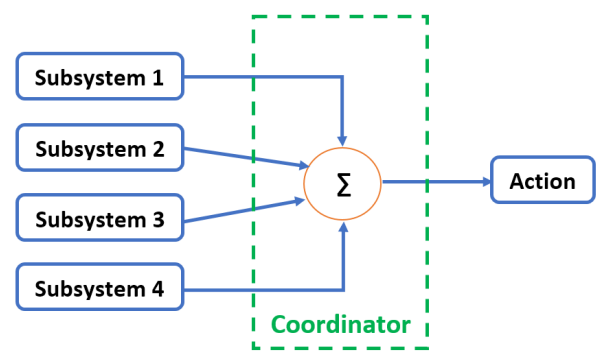

Fig. 3. Pure Subsumption coordination (adapted from [4]).

In this context, the work in [11] used Active Differential (AD), Electronic Stability Control (ESC), and Torque Vectoring (TV) to improve the vehicle lateral performances. A simple method based on prioritizing one system over another has been used. If the yaw torque demand can be satisfied by the AD, then the ESC and TV will not be activated. Otherwise, the rest of the yaw torque demand will be equally shared between the ESC and the TV systems. Both vehicle performance and safety have been improved within this method.

\section{B. Largest Modulus Activation}

In the largest modulus activation, several high level commands are considered, and the one with the highest modulus takes precedence over the rest (see Fig. 4).

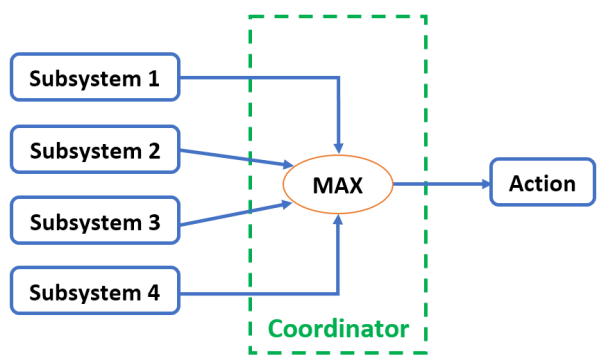

Fig. 4. Largest Modulus Activation coordination (adapted from [4]).

Both pure subsumption and largest modulus activation methods are characterized by modes switching. These switches 
generate undesirable transients that could destabilize the overall system. In this context, the Artificial Neural Network and the Fuzzy Logic Control were introduced.

\section{Artificial Neural Network}

The Artificial Neural Network consists of simple averaging or via a non-linear interpolation function weights (see Fig. 5).

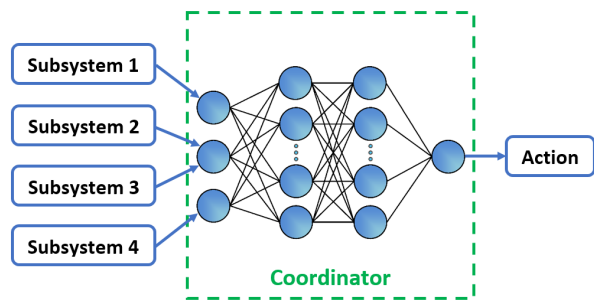

Fig. 5. Artificial Neural Network coordination (adapted from [4]).

These functions could be chosen to ensure smooth transitions between coordination modes, actuator saturation avoidance, etc. [12].

\section{Fuzzy Logic}

The Fuzzy Logic uses "easily understood" rule-based coordination functions (see Fig. 6).

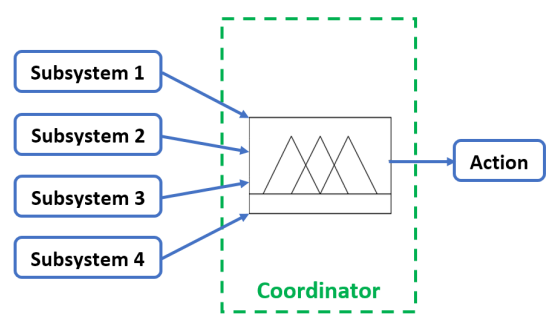

Fig. 6. Fuzzy Logic coordination (adapted from [4])

Here again, the highest level predominates but smooth transitions are ensured [4]. In [13] for example, a fuzzy logic scheme and weighting factors are used to coordinate the different systems. The controller agents computes combined control signals for the steering angle and the wheel torque depending on the targeted performances priority. The main advantage is the conflict mitigation, for example, when the braking controller has to track simultaneously the yaw rate reference and the longitudinal acceleration demand.

\section{E. Architecture's Compatibility}

From the examples in the previous subsections, it appears that the downstream coordination approach is more suitable for single objective coordination control. For multi-objective control, Other coordination algorithms could be cited as the Linear Quadratic Regulator (LQR), Lyapunov functions, sliding mode control, adaptive control, etc. These optimization methods have the major advantage of dynamic coordination, which ensure dynamic transitions. They offer possibilities for robust and fault-tolerant vehicle control systems without $a$ priori knowledge of anticipated failure modes [4]. Thus, the redundancy (that could be very expensive) only concerns the basic hardware needs. These methods are rather used in an upstream coordination approach.

\section{SySTEMS UPSTREAM COORDINATION}

Here, the coordination is carried out upstream the subsystems. The commands are distributed in a way to avoid the conflicts downstream the subsystems. A multivariable controller is placed between the driver/pilot commands and the chassis systems. The controller design is based on a coupled nonlinear vehicle model that give insights about the possible conflicts before reaching them. In [8], this approach is called "top-down approach". To illustrate this approach, we reproduce the structure given in [8] (Fig. 7).

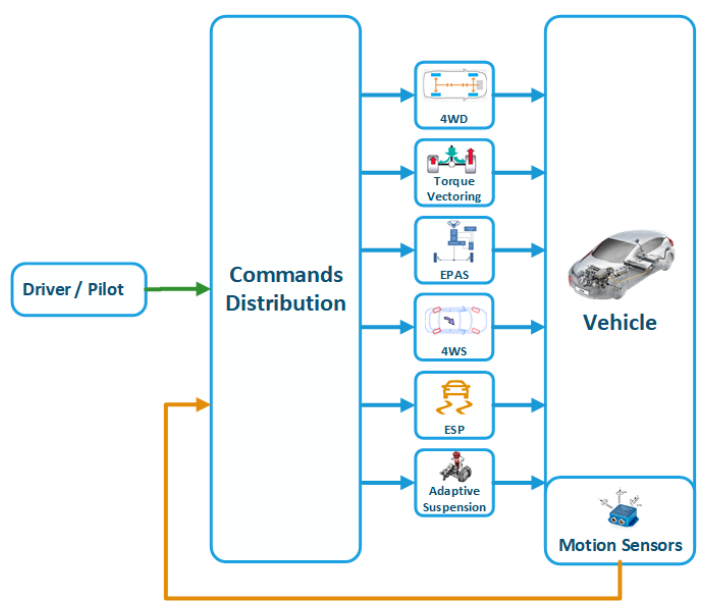

Fig. 7. Structure of the downstream coordination approach (adapted from [8]).

In accordance to this approach, three architectures have been distinguished in [4] and [9]: centralized control, supervisory control, and decentralized control. As we have mentioned, the decentralized control rather corresponds to downstream coordination. We present only the centralized control (A) and the supervisory control (B).

A third class could be distinguished, which is more of an extension of the supervisory control, called the multi-layer architecture (C).

\section{A. Centralized Control}

A central global controller is responsible of taking all the control decisions. In general, this controller follows the global multivariable control formalism as it has been realized in [14][17]. Fig. 8 illustrates this concept.

However, it has been pointed out in [18] that any desired fail-safe redundancy of micro-controllers or power converters increases rapidly the cost of the control components. For that matter, a distributed control method has been preferred, which can be assimilated to a supervisory control [4].

\section{B. Supervisory Control}

Supervisory control represents an intermediate between the centralized and decentralized control. Indeed, a supervisory 


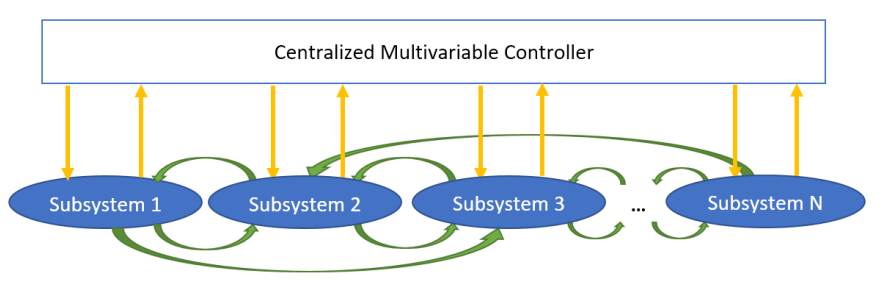

Fig. 8. Centralized control structure (adapted from [4]).

layer is added to a decentralized structure to add more information in the process. Fig. 9 illustrates this approach.

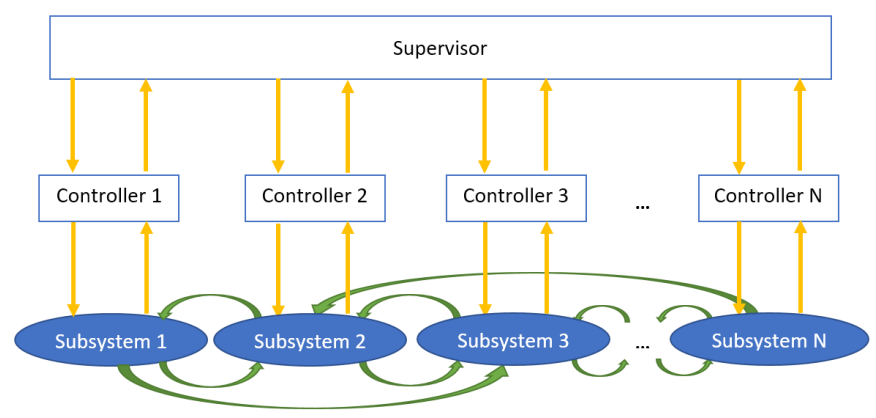

Fig. 9. Supervisory control structure (adapted from [4]).

Three advantages could be deduced from this architecture:

- Fault-tolerance: it ensures a minimum of operations safety even if the high-level controller fails,

- Extensibility: it can be evolved to a multi-layer hierarchical structure to add more functionalities,

- Modularity: it allows manufacturers and suppliers develop independently complementary control algorithms.

Using this structure, [10] used three main layers with two levels of abstraction:

1) Decision Layer: Identifies the current driving situation first, then decide how to coordinate the subsystems actions,

2) Control Layer: Transforms the control objectives generated by the Decision Layer to references for each of the local controllers,

3) Physical Layer: Contains simply the different actuators and sensors.

It should be noted that the decision layer plays a major role to ensure the overall system safety. It is responsible of two main tasks: classifying the current driving situation and deciding how coordination should be made. For example, in [10], a k-means data-based algorithm and a decision logic module based on a set of heuristic rules have been used ${ }^{1}$.

\section{The Multi-layer architecture}

For more flexibility and more comprehensive control, the functional requirements should rather be separated while ensuring a supervised control. Fig. 10 illustrates this method.

\footnotetext{
${ }^{1}$ Comparison of decision techniques is beyond the scope of this paper.
}

Each layer has a specific function [19]:

1) Layer 1: Generation of vehicle motion reference,

2) Layer 2: Decision on the control mode based on the vehicle state recognition,

3) Layer 3: Calculation of the generalized forces and moments at the vehicle's center of gravity through the high-level controllers,

4) Layer 4: Distribution of the commands to the available actuators in an optimal or sub-optimal way through control allocation logic,

5) Layer 5: Control of stand-alone subsystems to follow the commands that comes from the layer 4 ,

6) Layer 6: Execution of the various operations through smart actuators composed of low-level effectors (e.g. electric motor, hydraulic valve ...etc.) and their own controllers.

\section{Architecture's Compatibility}

This approach can be used for single objective coordination control. For example, lateral performances have been improved by integrating Active Front Steering (AFS) and the brakebased Dynamic Yaw Control (DYC). In this context, model predictive control [20], model-following control [21], and even fuzzy logic control in an upstream coordination structure [22], [23] were used. These studies showed not only improvement of lateral stability, but also manoeuvrability and agility were enhanced. However, the current cost of this architecture do not justify the achieved gains.

Most of interesting studies carried out using this approach concern the multiple objective coordination control. In this context, various integration methods have been adopted. For example, to deal with lateral and vertical integration, active suspension has been combined with brake-based control in [24]. A Linear Parameter Varying (LPV) design with faulttolerant control has been used. The commands distribution is made by using three weighting functions for lateral acceleration, heave acceleration, and suspension deflection. The results showed attractive improvements in rough surface conditions.

In the past years, control allocation techniques became more preponderant, e.g. [19], [25]-[27]. For example the authors of [26] used an Integrated Chassis Control (ICC) strategy to improve cornering performance in high speed by combining the ESC, the 4-Wheel Drive (4WD) and the Active Roll Control (ARC) systems. The control architecture is composed of thee parts: a supervisory controller that determines the target vehicle motions, the upper-level controller that calculates the target forces and moment and the lower-level controller that optimally distributes the actuator inputs. The same architecture has been adopted in [27]. The subsystems coordination algorithm uses restriction weights that changes according to the performances targeted. The investigations were carried out by a hardware-in-the-loop test rig, which demonstrated potential enhancement for different performances. Regarding the optimization methods, we can cite 3 approaches [28]: Update laws [29], Repeated optimization [30], [31] and Precomputed laws [32]. 


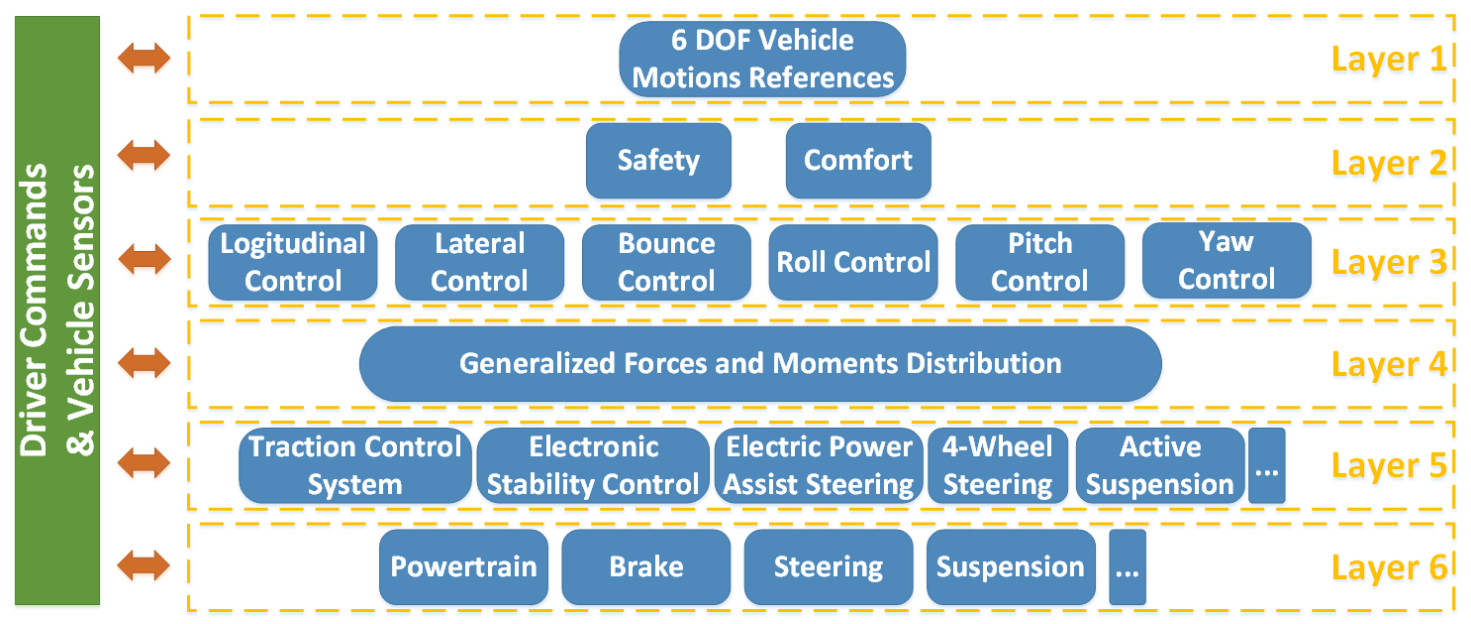

Fig. 10. Multi-layered control architecture [19].

However, as pointed out by [7], all the results are made by simulation only. There is a clear lack of experimental results and benchmark requirements that allow comparison between the different methods.

\section{COMPARISON AND Challenges}

\section{A. Relevance of the new classification}

We recall that two enriching reviews of integrated architectures have already been proposed in [4] and [7]. In [4], emphasis has been put on the architectures' topology. The authors pointed out two major extremes, the fully decentralized control and the fully centralized control, considering the supervisory control as an intermediate between them. This allowed the authors to highlight the benefits of a multi-layer architecture and to draw specific requirements related to the architecture topology: modularity, simplicity, fault-tolerance, and openness. However, no relations were proposed between the different structures and control objectives. As "simplicity" should be one of the architecture's requirements, a multi-layer architecture may seem exaggerated for single-objective control problems.

The authors of [7] focused rather on control objectives. Two classes have been proposed: single-criterion and multicriterion. They studied a large amount of examples for both classes, but conclusions were limited to the control methods and challenges. They particularly highlighted that most singlecriterion solutions are using rule-based methods (e.g. fuzzy logic control), while recent researches around multi-criterion control are using optimization methods, especially control allocation. Unlike [4], no discussion about the architectures topology is provided. We recall that fuzzy logic could be used downstream the stand-alone subsystems for single-objective control [8], or upstream the stand-alone subsystems for multiobjective control [22].

In order to gather the different stakeholders to start thinking on the standardization of integrated vehicle dynamics control architectures, we have to make a bridge between the two types of classifications. For this reason, we believe that the architecture topology should be related to the control objective. As we are interested in subsystems coordination, the topologies classification should be linked to the coordination layer position with respect to stand-alone subsystems. In this context, two approaches have been distinguished in [8]: the "bottom-up" approach (which is called in this paper downstream coordination) approach, and the "top-down" approach (referred to the upstream coordination). However, in [8], only the centralized control structure was given to the top-down approach. The concluding remarks were that the top-down approach is unfavourable from an industrial perspective for its difficulty and $\operatorname{cost}^{2}$. The bottom-up approach was then chosen and Fuzzy Logic with $\beta$-phase plane control was selected. The main differences that we provide with respect to [8] are a broader distinction of each approach and their suitability for control objectives. Therefore, the control designer would be able to automatically choose a standardized architecture depending on its objectives. The new classification proposed in this paper seems to be relevant within this framework. $U p$ stream or downstream coordination not only designate where, but also how the coordination is managed. This has allowed us to link each approach to control objectives. When the coordination is made downstream, rule-based control design is used. This technique can handle well single-objective control problems. As more interactions are added, it is hard to foresee the different couplings induced, so it is more complicated to formalize additional rules to achieve safe coordination. When the coordination is made upstream, the control design is based on a coupled vehicle model. Different interactions could be predicted. With an adapted optimization technique, multi-objective control problems could be handled.

\section{B. Comparison of the two approaches}

1) Complexity: The main advantage of the downstream approach is the low complexity as long as the number of interactions between competing systems are low. The design

\footnotetext{
${ }^{2}$ This has changed today.
} 
methodology consists on first studying the interactions between two or more systems and then establishing adequate rules to benefit from their potential synergies. This could be done for example by using a fuzzy logic approach or the $\beta$ phase plane control [8]. On the other hand, complexity is the main drawback of the upstream approach. Firstly, the coordination lies on a MIMO (Multiple Inputs Multiple Outputs) controller based on a coupled non-linear vehicle model. Then, optimization techniques are used to solve the problem, in realtime. However, the more complex the interaction between the competing systems get nonetheless with regards to emerging behaviour prediction, the more this upstream approach is pertinent.

2) Cost: Here, also the downstream approach is more attractive. In fact, without modifying the structure of the subsystem control logic, automobile manufacturers can proceed to bulk purchasing from OEMs taking advantage from the economy of scale. Moreover, the architecture does not require additional controllers, but simply a coordination strategy made downstream. Unfortunately, this is not the case for the upstream approach. Not only additional high-level controller(s) is needed, but it may also require additional sensors or estimators [42]. For these reasons, auto-makers are reluctant to implement this approach in real vehicles.

3) Potential: The downstream approach is based on "expert knowledge" methods [8]. This consists in using some preliminary use-case studies and control designers' expertise to develop arbitration strategies. This approach is not well formalized because, theoretically, we cannot cover all the possible use-cases. It is hard to measure this approach potential, so we are not able to know if optimal results are achieved, or at least if better results could be obtained. Another issue is the fact that the subsystems control laws may then be based on different reduced vehicle models ${ }^{3}$. For instance, the development of an Active Rear Steering (ARS) is based on just the simple bicycle model [14]. As a result, putting together different systems based on different behaviour models does not ensure proper operation of the overall vehicle system. In contrast, the upstream approach depicts mathematically the dynamic interactions, which is more suitable for numerical processes. Couplings can be quantified, so we can have some insights about the possibility of finding an optimum solution, a sub-optimal solution or no solution at all. The conflicts are rather prevented than mitigated. For this reason, upstream coordination techniques have more potential in handling multiobjective control problems.

\section{Summary}

It appears that a compromise should be made between complexity, cost, and potential. To avoid complexity, the control designer could prefer a downstream approach as long as the problem consists on a single-objective control. As more interactions are added, upstream approach becomes necessary for safety matters.

\footnotetext{
${ }^{3}$ This is certainly the case when produced by different suppliers but may also be the case for a single supplier.
}

Although the upstream approach may seem expensive, the ECUs have became faster, less cumbersome, and cheaper in the past years. Moreover, various algorithms have been developed and tested. According to [19], [33], [34]-[37], control allocation methods are more suitable for this problem, especially for over-actuated vehicles. These methods have been reviewed and compared in [30], [38]. Linear Programming (LP) and Quadratic Programming (QP), could be executed in a few milliseconds with a limited number of iterations, which real-time computations require. These advances may finally convince auto-mobile manufacturers to adopt this approach.

As the main aim of this paper is to invite auto-makers and suppliers to standardize one overall architecture, one approach should be put on the spotlight. With the arrival of autonomous vehicles, the virtual pilot should take into account multiple objectives at the same time. As we have mentioned, downstream coordination is more suitable for single objective control where the human pilot deals with the other performances. For example, in a high speed cornering manoeuvre, the driver controls the longitudinal acceleration while the active steering could be combined with the brake-based yaw control to control the lateral dynamics and stabilize the vehicle. In autonomous driving, these two objectives should be fulfilled by the virtual pilot. Interactions should be predicted, and conflicts should be avoided rather than mitigated. Consequently, as we are moving towards the autonomous driving, the upstream coordination approach will become necessary.

For this reason, vehicle manufacturers and suppliers should prepare a common overall architecture. This architecture should have the following criteria:

- Adaptability to face environment changes and drivers behaviors [2],

- Fault-tolerance to propose some degraded modes and ensure a minimum of safety,

- Dynamic reconfiguration to ensure soft switching and prevent loss of stability [6],

- Extensibility to rapidly insert additional technologies without redesigning the whole architecture [2],

- Modularity to ensure flexibility,

- Openness to support various systems from different sources without jeopardizing the intellectual property rights of the different stakeholders [2].

As a consequence, the multi-layer architecture with the control allocation method seems to be a better choice to fulfill these criteria [25]-[28], [39]-[43]. For example, the advanced approach presented in [40]-[43] uses vehicle state estimator based on extended Kalman filter, high-level controller of vehicle general motion, middle-level control allocation and lower-level controllers for each subsystem as the Fig.11 shows.

It should be noted that this architecture has been validated in a Hardware-In-the-Loop (HIL) procedure [42]. For all these reasons, we believe that this architecture is worth investigating and should be implemented in a real vehicle. 


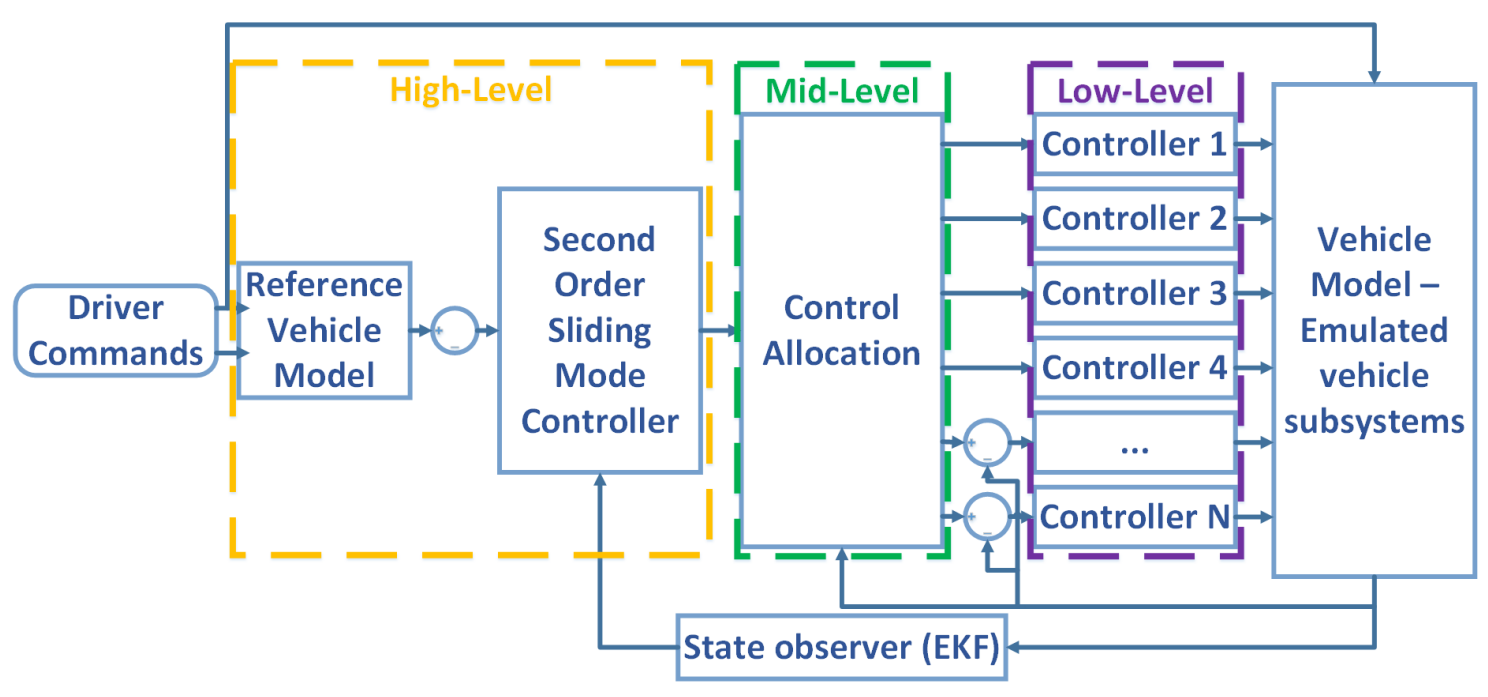

Fig. 11. General architecture of Integrated Motion Control [42].

\section{Open challenges}

1) Real vehicle implementation: As we mentioned, the first challenge is to be able to implement this architecture in a real vehicle. Real-time computation of an optimization method is usually a challenging task. In Integrated Vehicle Dynamics Dynamics Control, the challenge gets bigger as the coordination technique is located in an inner loop. Consequently, a higher rate is required (around $100 \mathrm{~Hz}$ according to [30]). Control allocation with linear or quadratic programming could be the solution [30], [38]. Another issue is to bring together manufacturers and suppliers to collaborate. That goes beyond the scope of our work. Nevertheless, attractive results for both sides could be the first step.

2) “Adaptability": As long as over-actuated systems are concerned, multiple solutions could be found for an optimization problem. Secondary objectives could therefore be achieved. Allocation can be used to favour one solution over another according to the desired behaviour of the vehicle. Consequently, different "feelings" can be generated to realize the same manoeuvre. While controlling the yaw rate using the 4-Wheel Drive system could give the vehicle a sporty behaviour, using the 4-Wheel Steering system for the same manoeuvre could rather give a comfortable behaviour. This is of major importance for autonomous vehicles where the challenge is not only the trajectory following but also how the vehicle follows this trajectory. Control allocation introduces new opportunities to make drivers accept autonomous vehicles. Using specific weighting functions to favour different subsystems combinations could be used to generate feelings of comfort and security, and therefore make drivers trust more their vehicles. However, the intra and inter-individual variability between the drivers is also a challenge. Motions that generate excitement for some people could generate fear among others. So allocation should be adaptable and change over time and maybe even learn from its driver's preferences. Evolutionary algorithms and artificial intelligence could be an interesting approach to investigate in this field too.

\section{COnClusion And Future WORKS}

In this paper, a new classification of integrated vehicle dynamics control architectures has been proposed. Two major classes related to the coordination logic have been outlined: the downstream coordination architecture and the upstream coordination architecture. These two classes have been compared. The downstream coordination requires less cost and less complexity but it is limited to single-objective control coordination. In contrast, the upstream coordination can handle multi-objective control coordination but it is more complex and requires more costs. These last drawbacks could be overcame by the advances made in electronic and software engineering.

The literature shows that the integrated vehicle dynamics control architectures are still an object of research. There is a clear lack of benchmark standards and common test procedures to validate the integrated subsystems coordination methods. This paper aims to invite auto-mobile manufacturers and suppliers to adopt an upstream approach and to standardize its structure.

We recognize that more evidence are needed to convince the different stakeholders to favour one architecture over another. That is why our future works will concern the development of the multi-layer architecture with control allocation methods and their comparison with the downstream approach.

The first step of control synthesis is modelling. Regarding ground vehicles, tires are the sole effectors. Distribution of control commands is mainly constrained by tires' potential. As far as combined slip is concerned, this potential varies. Tires' stiffness and maximum efforts should be updated on-line. In this way, we can favour the tires with greater potential. For these reasons, a new tire model is under development. This model is linear in order to facilitate control synthesis, and parameter varying to depict combined slip. 


\section{ACKNOWLEDGEMENT}

The authors would like to thank Dr. Xavier Mouton and Dr. Didier Martinez from the Group Renault for their enriching discussions about today's automotive industry challenges.

\section{REFERENCES}

[1] B. Heibing and M. Ersoy (Eds.), "Chassis Handbook - Fundamentals, Driving Dynamics, Components, Mechatronics, Perspectives," Vieweg+Teubner Verlag - Springer Fachmedien Wiesbaden GmbH 2011.

[2] E. Coelingh, P. Chaumette and M. Andersson, "Open-interface definitions for automotive systems - Application to a Brake by Wire System," in SAE 2002 World Congress, 2002-01-0267.

[3] Y. Zhang and J. Jiang, "Bibliographical review on reconfigurable faulttolerant control systems," in Annual Reviews in Control, 32(2), 229-252, 2008

[4] T. Gordon, M. Howell and F. Brandao, "Integrated control methodologies for road vehicles," in Vehicle Syst. Dyn., Int. J. Vehicle Mech. Mobility, vol. 40, nos. 1-3, pp. 157-190, 2003.

[5] N. Navet and F. Simonot-Lion, "Automotive embedded systems handbook," CRC press, 2008.

[6] L. Wills, S. Kannan, S. Sander, M. Guler, M., B. Heck, J. Prasad, D. Schrage and G. Vachtsevanos, "An open platform for reconfigurable control," in IEEE Control Systems Magazine, 21(3), (2001), pp.49-64.

[7] V. Ivanov and D. Savitski, "Systematization of Integrated Motion Control of Ground Vehicles," in IEEE Access, vol. 3, no. , pp. 2080-2099, 2015.

[8] M. A. Selby, "Intelligent Vehicle Motion Control," PhD thesis, University of Leeds, Feb. 2003.

[9] N.A. Duffie, R. Chitturi and J. Mou., "Fault-tolerant heterarchical control of heterogeneous manufacturing system entities," in Journal of Manufacturing Systems, 7 (1988), pp.315-328.

[10] C.A. Vivas-Lopez, J.C. Tudon-Martinez, D. Hernandez-Alcantara and R. Morales-Menendez, "Global Chassis Control System Using Suspension, Steering, and Braking Subsystems," in Mathematical Problems in Engineering, vol. 2015, Article ID 263424, 18 pages, 2015.

[11] M. Velardocchia and A. Vigliani, "Control systems integration for enhanced vehicle dynamics," in The Open Mech. Eng. J., vol. 7, 2013 pp. $58-69$

[12] C. O. Nwagboso, X. Ouyang, and C. Morgan, "Development of neuralnetwork control of steer-by-wire system for intelligent vehicles," in Heavy Vehicle Systems, vol. 9, 2002, pp. 1-26.

[13] J.-X. Wang, N. Chen, D.-W. Pi and G.-D. Yin, "Agent-based coordination framework for integrated vehicle chassis control," in Proc. Inst. Mech. Eng., D, J. Automobile Eng., vol. 223, no. 5, pp. 601-621, 2009.

[14] S. Brennan and A. Alleyne, "Integrated vehicle control via coordinated steering and wheel torque inputs," in American Control Conference, (2001), pp.7-12.

[15] M. Nagai, S. Yamanaka and Y. Hirano, "Integrated control of active rear wheel steering and yaw moment control using braking forces," in JSME International Journal, 42 (2) Series C (1999), pp.301-308.

[16] M. Harada and H. Harada, "Analysis of lateral stability with integrated control of suspension and steering systems," in JSAE Review, 20 (1999), pp. $465-470$

[17] M. Nagai, Y. Hirano and S. Yamanaka, "Integrated robust control of active rear wheel steering and direct yaw moment control," in Vehicle System Dynamics, 28 (1998), pp.416-421.

[18] N.A. Kelling and W. Heck, "The BRAKE project centralized versus distributed redundancy for brake-by-wire systems," in SAE 2002 World Congress, (2002) 2002-01-0266.

[19] A. Soltani, "Low Cost Integration of Electric Power-Assisted Steering (EPAS) with Enhanced Stability Program (ESP)," PhD thesis, Cranfield University, 2014.

[20] P. Falcone, F. Borrelli, H. E. Tseng, J. Asgari and D. Hrovat, "Integrated braking and steering model predictive control approach in autonomous vehicles," in Proc. 5th IFAC Symp. Adv. Automot. Control, Seascape Resort, CA, USA, 2007, pp. 273-278.

[21] M. Abe and O. Mokhiamar, "An integration of vehicle motion controls for full drive-by-wire vehicle," in Proc. Inst. Mech. Eng., K, J. Multi-Body Dyn., vol. 221, no. 1, pp. 116-127, 2007.
[22] M. J. L. Boada, B. I. Boada, A. Muoz and V. Daz, "Integrated control of front-wheel steering and front braking forces on the basis of fuzzy logic," Proc. Inst. Mech. Eng., D, J. Automobile Eng., vol. 220, no. 3, pp. 253-267, 2006

[23] Y. Hou, J. Zhang, Y. Zhang and L. Chen, "Integrated chassis control using ANFIS," in Proc. IEEE Int. Conf. Autom. Logistics, Qingdao, China, Sep. 2008, pp. 1625-1630

[24] P. Gspr, Z. Szab and J. Bokor, "Active suspension in integrated vehicle control," in Switched Systems, J. Kleban, Ed. Rijeka, Croatia: InTech, 2009.

[25] G. Knobel, A. Pruckner and T. Bunte, "Optimized force allocation - A general approach to control and to investigate the motion of over-actuated vehicles," in Proc. 4th IFAC Symp. Mechatronic Syst., Heidelberg, Germany, 2006, pp. 366-371.

[26] H. Heo, E. Joa, K. Yi and K. Kim, "Integrated chassis control for enhancement of high speed cornering performance," in SAE Int. J. Commercial Vehicles, vol. 8, no. 1, pp. 102-109, 2015.

[27] B. Shyrokau, D. Wang and M. Lienkamp, "Integrated vehicle dynamics control based on control allocation with subsystem coordination," in Proc. 23rd IAVSD Int. Symp. Dyn. Vehicles Roads Tracks, Qingdao, China, 2013, pp. 1-10.

[28] E.H. van den Berg, "Global chassis control and braking control using tyre forces measurement". TU Delft, Delft University of Technology, 2011.

[29] M. Gerard, "Optimal Control Allocation on Over-Actuated Vehicles Maximizing vehicle performance and safety through advanced vehicle control strategies". Delft Center for Systems and Control (DCSC), Delft University of Technology, August 10, 2016.

[30] M. Bodson, "Evaluation of optimization methods for control allocation," in Journal of Guidance, Control, and Dynamics, vol. 25, no. 4, pp. 703711, 2002.

[31] O. Hrkegrd, "Dynamic control allocation using constrained quadratic programming," in Journal of Guidance, Control, and Dynamics, vol. 27, no. 6, pp. 10281034, 2004

[32] F. Borrelli, A. Bemporad, M. Fodor and D. Hrovat, "An mpc/hybrid system approach to traction control," in IEEE Transactions on Control Systems Technology, vol. 14, pp. 541552, May 2006.

[33] W. Chen, H. Xiao, L. Liu, J. W. Zu and H. Zhou, "Integrated control of vehicle system dynamics: Theory and experiment," in Advances in Mechatronics, H. Martnez-Alfaro, Ed. Rijeka, Croatia: InTech, 2011

[34] J. Tjnns, "Nonlinear and Adaptive Dynamic Control Allocation," Norway: NTNU, Trondheim, 2008.

[35] Y. Seongiin, "Coordinated Control with Electronic Stability Control and Active Steering Devices," in Journal of Mechanical Science and Technology, Vol 29 (12) (2015) 54095416.

[36] H. Zhao, B. Gao1, B. Ren, H. Chen, and W. Deng, "Model Predictive Control Allocation for Stability Improvement of Four-Wheel Drive Electric Vehicles in Critical Driving Condition," in IET Control Theory Appl., 2015, Vol. 9, Iss. 18, pp. 26882696

[37] Z. Shuai, H. Zhang, J. Wang, J. Li, M. Ouyang, "Lateral motion control for four-wheel-independent-drive electric vehicles using optimal torque allocation and dynamic message priority scheduling," in Control Engineering Practice, Vol. 24, March 2014, pp. 5566.

[38] T. A. Johansen and T. I. Fossen, "Control Allocation - A survey," in Automatica, Vol. 49, Issue 5, May 2013, Ppp. 10871103.

[39] P. Reinold and A. Traechtler, "Closed-loop control with optimal tireforce distribution for the horizontal dynamics of an electric vehicle with single wheel chassis actuators," in Proc. Amer. Control Conf. (ACC), Washington, DC, USA, Jun. 2013, pp. 2159-2164.

[40] B. Shyrokau and D. Wang, "Control allocation with dynamic weight scheduling for two-task integrated vehicle control," in Proc. 11th Int Symp. Adv. Vehicle Control, Seoul, Korea, 2012, pp. 1-6.

[41] B. Shyrokau and D. Wang, "Coordination of steer angles, tyre inflation pressure, brake and drive torques for vehicle dynamics control," in $S A E$ Int. J. Passenger Cars-Mech. Syst., vol. 6, no. 1, pp. 241-251, 2013.

[42] L. Heidrich, B. Shyrokau, D. Savitski, V. Ivanov, K. Augsburg and D. Wang, "Hardware-in-the-loop test rig for integrated vehicle control systems," in Proc. 7th IFAC Symp. Adv. Automot. Control, Tokyo, Japan, 2013, pp. 683-688.

[43] B. Shyrokau, D. Wang, D. Savitski, K. Hoepping and V. Ivanov, "Vehicle motion control with subsystem prioritization," in Mechatronics, pp. 1-12, Dec. 2014. 